Research, Society and Development, v. 9, n. 8, e409985102, 2020

(CC BY 4.0) | ISSN 2525-3409 | DOI: http://dx.doi.org/10.33448/rsd-v9i8.5102

Análise dos métodos da norma ABNT NBR 15527/2007 para o dimensionamento de reservatórios de águas pluviais em municípios do semiárido pernambucano no Brasil Analyzes methods of ABNT NBR 15527/2007 for sizing rainwater water reservoirs in municipalities in the semiarid pernambucano in Brazil

Analiza métodos de ABNT NBR 15527/2007 para dimensionar reservas de agua de lluvia en municipios en el semiárido pernambucano en Brasil

Recebido: 29/05/2020 | Revisado: 27/06/2020 | Aceito: 29/06/2020 | Publicado: 11/07/2020

Lucas Vinícius dos Santos Ferreira ORCID: https://orcid.org/0000-0003-4626-8849

Universidade Federal de Pernambuco, Brasil E-mail: lucasvferreira96@gmail.com José Martins de França Neto ORCID: https://orcid.org/0000-0002-4094-6138 Universidade Federal de Pernambuco, Brasil E-mail: jmfrancaneto@gmail.com Larissa Virgínia da Silva Ribas ORCID: https://orcid.org/0000-0003-4910-3644 Universidade Federal da Bahia, Brasil E-mail: larissa.vribas@gmail.com Lucas Ravellys Pyrrho de Alcântara ORCID: https://orcid.org/0000-0002-7259-8510

Universidade Federal de Pernambuco, Brasil E-mail: ravellyspyrrho@gmail.com Severino Martins dos Santos Neto ORCID: https://orcid.org/0000-0003-2940-2129 Universidade Federal de Pernambuco, Brasil E-mail: martinsdsn@gmail.com Antonio Celso Dantas Antonino ORCID: https://orcid.org/0000-0002-4120-9404

Universidade Federal de Pernambuco, Brasil E-mail: acdantonino@gmail.com 


\title{
Artur Paiva Coutinho
}

ORCID: https://orcid.org/0000-0002-0644-0037

Universidade Federal da Pernambuco, Brasil

E-mail: arthur.coutinho@yahoo.com.br

\section{Resumo}

O semiárido brasileiro apresenta grandes desafios para o abastecimento e gerenciamento dos recursos hídricos. Como alternativa, sistemas de aproveitamento de águas pluviais (SAAP) são amplamente utilizados. A ABNT NBR 15527/2007 normatiza seis métodos de dimensionamento de reservatórios, no qual suas aplicações estão relacionadas às especificidades climáticas das regiões. Esse trabalho efetua o estudo de aplicabilidade dos métodos normatizados para os municípios de Caruaru, Bezerros e Gravatá, localizados na região semiárida do estado de Pernambuco. O objetivo é avaliar o método mais apropriado para cada município, avaliando a garantia do abastecimento e a viabilidade financeira de execução. Para isso, foi estabelecida uma residência hipotética de referência com área coberta de $160 \mathrm{~m} 2$. O regime pluviométrico foi determinado conforme uma série histórica de 4 anos para cada município. A viabilidade econômica foi verificada com base nos custos executivos dos reservatórios e a economia financeira com base em tarifas locais. Como resultado, foi visto que os métodos da Simulação e Prático Australiano são os mais indicados, por oferecerem uma maior taxa de confiança quanto ao atendimento à demanda, bem como, por também possuírem estimativa de retorno do valor investido relativamente rápido, comparado a outros métodos.

Palavras-chave: Escassez hídrica; Sustentabilidade; Cisternas; Viabilidade econômica.

\begin{abstract}
The Brazilian semiarid region presents great challenges for the supply and management of water resources. Alternatively, rainwater harvesting systems (RWHS) are widely used. However, there are uncertainties about the methods of dimensioning the reservoirs of RWHS. ABNT NBR 15527/2007 standardizes six methods of reservoir dimensioning, in which their applications are related to the climatic specificities of the regions. Therefore, this work proposes the study of the applicability of standardized methods for the municipalities of Caruaru, Bezerros and Gravatá in the semiarid region of the state of Pernambuco. The
\end{abstract}


objective is to evaluate the most appropriate method for each municipality, evaluating the guarantee of supply and the financial viability of execution. For this purpose, a hypothetical residence with a covered area of $160 \mathrm{~m} 2$ was established. The pluviometric regime was determined according to a historical series of 4 years for each municipality. Economic viability was verified based on the costs for execution of the reservoirs and financial savings based on local tariffs. As a result, it was seen that the Simulation and Australian Practical methods are the most proper, as they offer a higher rate of certainty in meeting demand, as well as because they also have a relatively quick estimated return on investment, compared to other methods.

Keywords: Water scarcity; Sustainability; Cisterns; Economic viability.

\section{Resumen}

La región semiárida brasileña presenta grandes desafíos para el suministro y la gestión de los recursos hídricos. Alternativamente, los sistemas de recolección de agua de lluvia (SAAP) son ampliamente utilizados. ABNT NBR 15527/2007 estandariza seis métodos de dimensionamiento de yacimientos, en los cuales sus aplicaciones están relacionadas con las especificidades climáticas de las regiones. Este trabajo lleva a cabo el estudio de la aplicabilidad de los métodos estandarizados para los municipios de Caruaru, Bezerros y Gravatá, ubicados en la región semiárida del estado de Pernambuco. El objetivo es evaluar el método más apropiado para cada municipio, evaluando la garantía de suministro y la viabilidad financiera de la ejecución. Para este propósito, se estableció una hipotética residencia de referencia con un área cubierta de $160 \mathrm{~m} 2$. El régimen pluviométrico se determinó según una serie histórica de 4 años para cada municipio. La viabilidad económica se verificó en función de los costos ejecutivos de los embalses y los ahorros financieros en función de las tarifas locales. Como resultado, se vio que los métodos prácticos y de simulación de Australia son los más adecuados, ya que ofrecen una mayor tasa de confianza para satisfacer la demanda, así como porque también tienen un retorno de la inversión estimado relativamente rápido, en comparación con otros métodos.

Palabras clave: Escasez de agua; Sustentabilidad; Cisternas; Viabilidad económica.

\section{Introdução}

As regiões semiáridas brasileiras enfrentam crescentes desafios associados ao abastecimento hídrico, onde a implementação de políticas públicas e alternativas relacionadas 
à gestão hídrica são fundamentais (Lee et al., 2016; Pereira et al., 2018). A mesorregião do Agreste do estado de Pernambuco apresenta clima semiárido, com baixa disponibilidade hídrica subterrânea e colapso de seus principais mananciais de abastecimento hídrico. Em consequência, vários municípios sofrem com sistemas de rodízio de abastecimento, atravessando longos períodos sem acesso regular a água. Nesse contexto, fontes alternativas de abastecimento como sistemas de aproveitamento de águas pluviais (Sistemas AAP) tornam-se uma das principais alternativas para as épocas mais secas do ano.

A principal premissa de sistemas AAP é a conservação e redução do consumo de água potável aplicada para fins não nobres, significando também economia nos custos com abastecimento (Duarte et al., 2015; Fonseca et al., 2017). Essa prática é considerada de baixo custo e de fácil acesso a população, sendo empregada em zona rural para uso doméstico, irrigação e criação de animais (Campisano et al., 2017; Feitosa et al., 2018; Teston et al., 2018). Em ambientes urbanos sistemas AAP também favorecem a redução do escoamento superficial, funcionando como medida não estrutural no sistema de drenagem urbana (Campisano et al., 2014; KuchinskieGastaldini, 2016; Haque et al., 2016; Palla et al., 2017; Moura et al., 2018; Neves Filho et al., 2019).

Os sistemas AAP consistem em três componentes principais: captação, transporte e reservatório. Lima et al., (2017) ressaltam que o dimensionamento da capacidade do reservatório é considerado o ponto mais crítico e oneroso na implantação do sistema. Nesse sentido, a concepção do dimensionamento do reservatório compreende aspectos como a área de captação, o histórico pluviométrico local e a demanda de água de chuva requerida, que se refere ao volume de água potável que será substituída por água pluvial na edificação. Deve-se considerar também o custo da execução do projeto e o retorno do investimento. $\mathrm{O}$ dimensionamento correto do reservatório pode evitar excesso de gastos com superdimensionamento ou ineficiência causada pelo subdimensionamento (SOUZA et al., 2016).

No Brasil, a ABNT NBR 15527/2007 fornece seis metodologias diferentes para o cálculo do volume do reservatório: Método de Rippl, Método da Simulação, Método de Azevedo Neto, Método Prático Alemão, Método Prático Inglês e Método Prático Australiano. Entretanto, Pelak \& Porporato (2016) ressaltam que esses métodos apresentam vários inconvenientes, como especificidades para regiões particulares, dependência de otimização numérica e falhas em considerar os custos do sistema. A viabilidade do método deve considerar aspectos técnicos e econômicos específicos de cada região. De acordo com Dornelles et al. (2010), tais metodologias podem apresentar uma vasta gama de resultados 
para os mesmos cenários, dificultando a decisão de escolha do método adequado.

Com a ampla variabilidade destacada, aumentam as dúvidas dos projetistas de qual metodologia adotar, levando a possíveis falhas de abastecimento e desperdício de volumes de água. Conforme Dornelles et al. (2010) a seleção da técnica é fortemente influenciada pelo seu grau de facilidade no processo de cálculo. Estes autores destacam a importância de efetuar uma investigação comparativa, antes de escolher o método de dimensionamento.

Nesse sentido, alguns estudos realizaram a comparação dos métodos de dimensionamento da NBR 15527/07. Por exemplo, Dornellas et al. (2010) avaliaram os métodos da NBR 15527/07, visando propor um coeficiente de redução da precipitação média anual evitando o comportamento deficitário do reservatório. Brandão e Marcon (2018) analisaram os métodos de dimensionamento para diferentes cenários de captação da chuva, realizando de simulações diárias dos reservatórios e pelo cálculo da garantia de abastecimento. Fonseca e Menezes Filho (2019) consideraram uma residência unifamiliar hipotética em três capitais brasileiras e destacam que dentre os métodos, os de simulação propiciaram valores seguros de acordo com um índice de desempenho. Lima et al. (2017) aplicaram os métodos normatizados em uma análise de viabilidade econômica na implantação de um sistema de AAP em uma escola na cidade do Recife-PE. Na qual o período de retorno do investimento foi de 6 anos e 25 dias substituindo 59,6\% da água potável pela pluvial.

Sendo assim, no intuito de colaborar para a compreensão dos métodos de cálculo de volume de reservatório. E melhorar o conhecimento de soluções eficazes no combate ao desabastecimento de água na região semiárida do estado de Pernambuco. Esse trabalho objetiva avaliar as diferentes metodologias para o dimensionamento de reservatórios de captação de água de chuva apresentadas pela ABNT NBR 15527/2007, em três cidades do agreste pernambucano, avaliando a viabilidade econômica para construção do reservatório.

\section{Metodologia}

\section{Classificação da pesquisa}

O presente estudo pode ser classificado como estudo de caso, conforme Pereira et al. (2018), por se tratar de uma análise detalhada de uma condição especial. No caso, uma região de estudo específica. Além disso, conforme os mesmos autores, essa pesquisa foi desenvolvida conforme o método quantitativo, por apresentar uma coleta de dados quantitativos por meio de medições de grandezas e seus resultados serem analisados por meio 
de técnicas matemáticas.

\section{Região de estudo}

A presente pesquisa foi desenvolvida na mesorregião do Agreste do estado de Pernambuco, nordeste brasileiro. Para o qual, foram selecionados três municípios: Caruaru, Bezerros e Gravatá, conforme apresentado na Figura 1.

Figura 1 - Mapa de situação dos municípios estudados em relação ao agreste de Pernambuco e Nordeste Brasileiro.
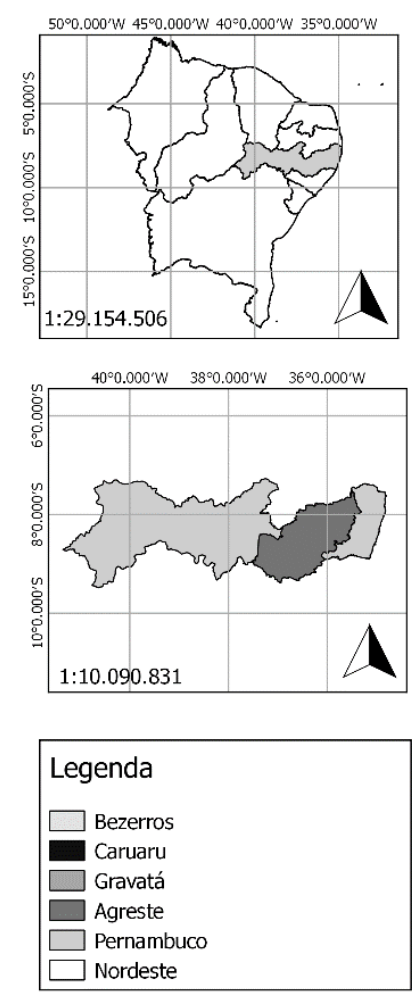

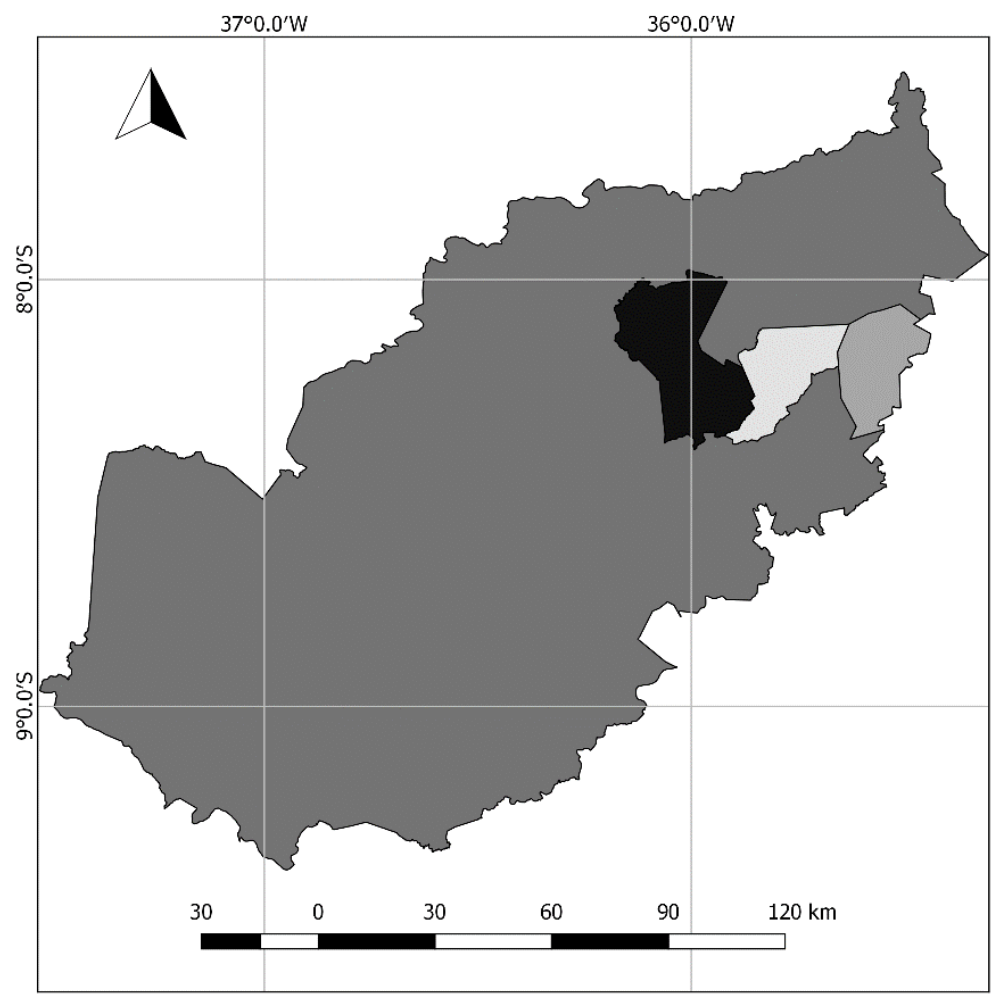

Fonte: Os Autores (2020).

O clima do agreste pernambucano é classificado como semiárido BSh (clima das estepes quentes de baixa latitude e altitude), segundo o modelo de Köppen. Na Tabela 1 são apresentados os dados referentes ao regime pluviométrico (médias de precipitações mensais e anuais) dos municípios em estudo, para uma série histórica de 4 anos (período que compreende os anos entre 2013 e 2016). Os dados coletados e tratados referem-se aos postos pluviométricos pertencentes à Agência Pernambucana de Águas e Climas, de nº 211, 67 e 58, para Caruaru, Bezerros e Gravatá, respectivamente. 
(CC BY 4.0) | ISSN 2525-3409 | DOI: http://dx.doi.org/10.33448/rsd-v9i8.5102

Tabela 1 - Médias mensais e anuais dos índices pluviométricos para os municípios de Caruaru, Bezerros e Gravatá da série história de 2013 a 2016.

\begin{tabular}{lccccccccccccc}
\hline Município & Jan & Fev & Mar & Abr & Mai & Jun & Jul & Ago & Set & Out & Nov & Dez & Anual \\
\hline Bezerros & 31,2 & 19,0 & 66,8 & 41,1 & 61,0 & 66,3 & 68,5 & 20,6 & 13,0 & 18,1 & 5,5 & 29,6 & 440,6 \\
Caruaru & 23,8 & 23,8 & 29,4 & 51,6 & 43,8 & 68,9 & 61,7 & 21,4 & 20,7 & 13,1 & 24,0 & 30,9 & 413,2 \\
Gravatá & 35,6 & 32,4 & 54,0 & 51,5 & 89,8 & 93,5 & 86,6 & 37,0 & 23,0 & 25,0 & 7,4 & 35,8 & 571,5
\end{tabular}

Fonte: APAC (2020).

\section{Área de captação e demanda de águas pluviais}

Para esse estudo de caso, foi estabelecida uma residência unifamiliar hipotética como referência, com área coberta de $160 \mathrm{~m}^{2}$ e 4 habitantes. A planta de coberta pode ser visualizada na Figura 2.

Figura 2 - Planta de cobertura da edificação em estudo, correspondendo a área de captação de águas pluviais.

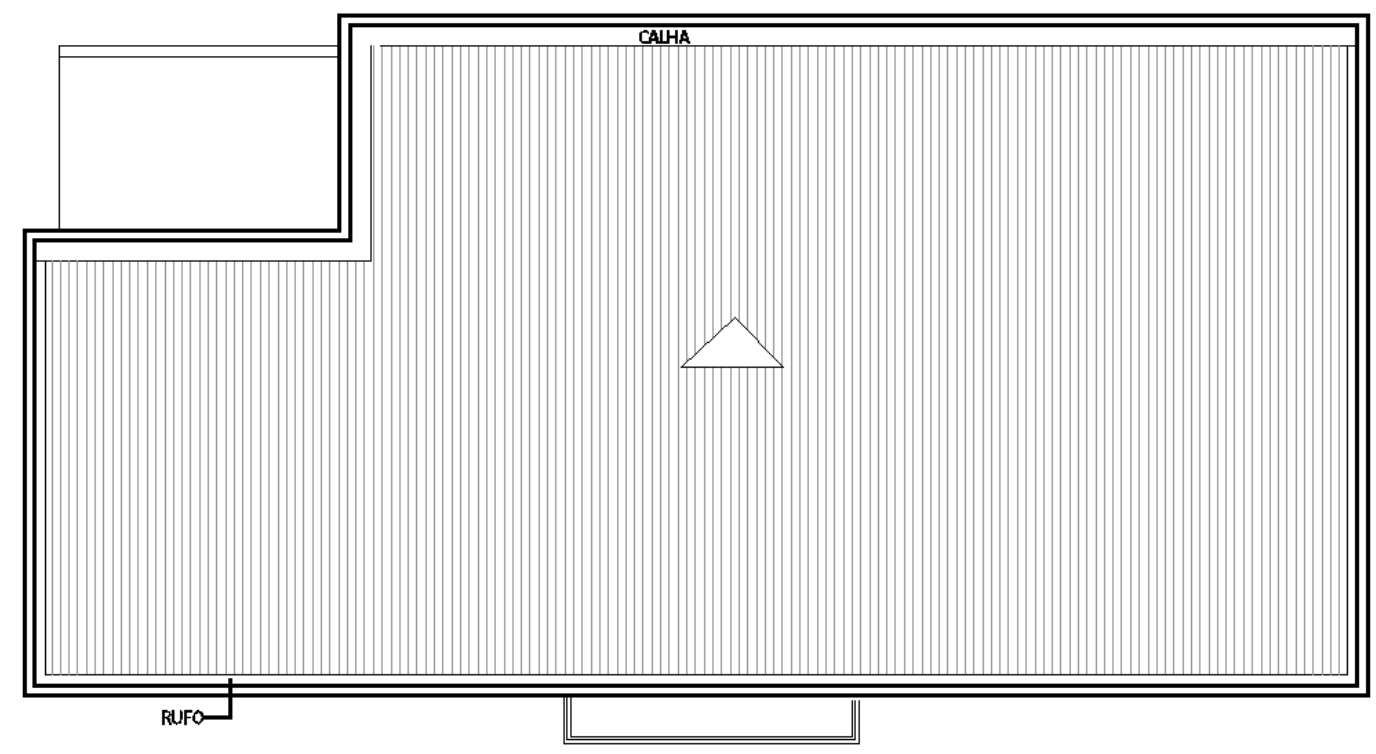

PLANTA DE COBERTA

ÁREA DA COBERTA $=160 \mathrm{~m}^{2}$

Fonte: Os Autores (2020).

O volume de água captado foi considerado apenas para descargas de vaso sanitários.

Sendo assim, foi considerada a utilização de uma bacia sanitária com caixa acoplada de 0,006 
Research, Society and Development, v. 9, n. 8, e409985102, 2020

(CC BY 4.0) | ISSN 2525-3409 | DOI: http://dx.doi.org/10.33448/rsd-v9i8.5102

$\mathrm{m}^{3}$. Foi considerado que cada habitante efetuou quatro acionamentos na descarga por dia, gerando um consumo de $0,096 \mathrm{~m}^{3} / \mathrm{dia}$ ou $2,88 \mathrm{~m}^{3} / \mathrm{mês}$. Foi adicionado $5 \%$ de perdas por vazamento, totalizando um consumo de $3 \mathrm{~m}^{3} / \mathrm{mês}$. Esse consumo é a demanda admitida para a captação no dimensionamento do Sistema AAP.

\section{Dimensionamento do reservatório}

De posse dos dados pluviométricos e considerando a área de coleta de $160 \mathrm{~m}^{2}$ foi possível calcular o volume de armazenamento dos reservatórios previstos para os três municípios. Todos os métodos utilizados para o dimensionamento dos reservatórios constam na ABNT NBR 15527/2007. As equações e as variáveis pertinentes de cada método são apresentadas na Tabela 2 .

Tabela 2 - Métodos da ABNT NBR 15527/2007 utilizados no dimensionamento do reservatório

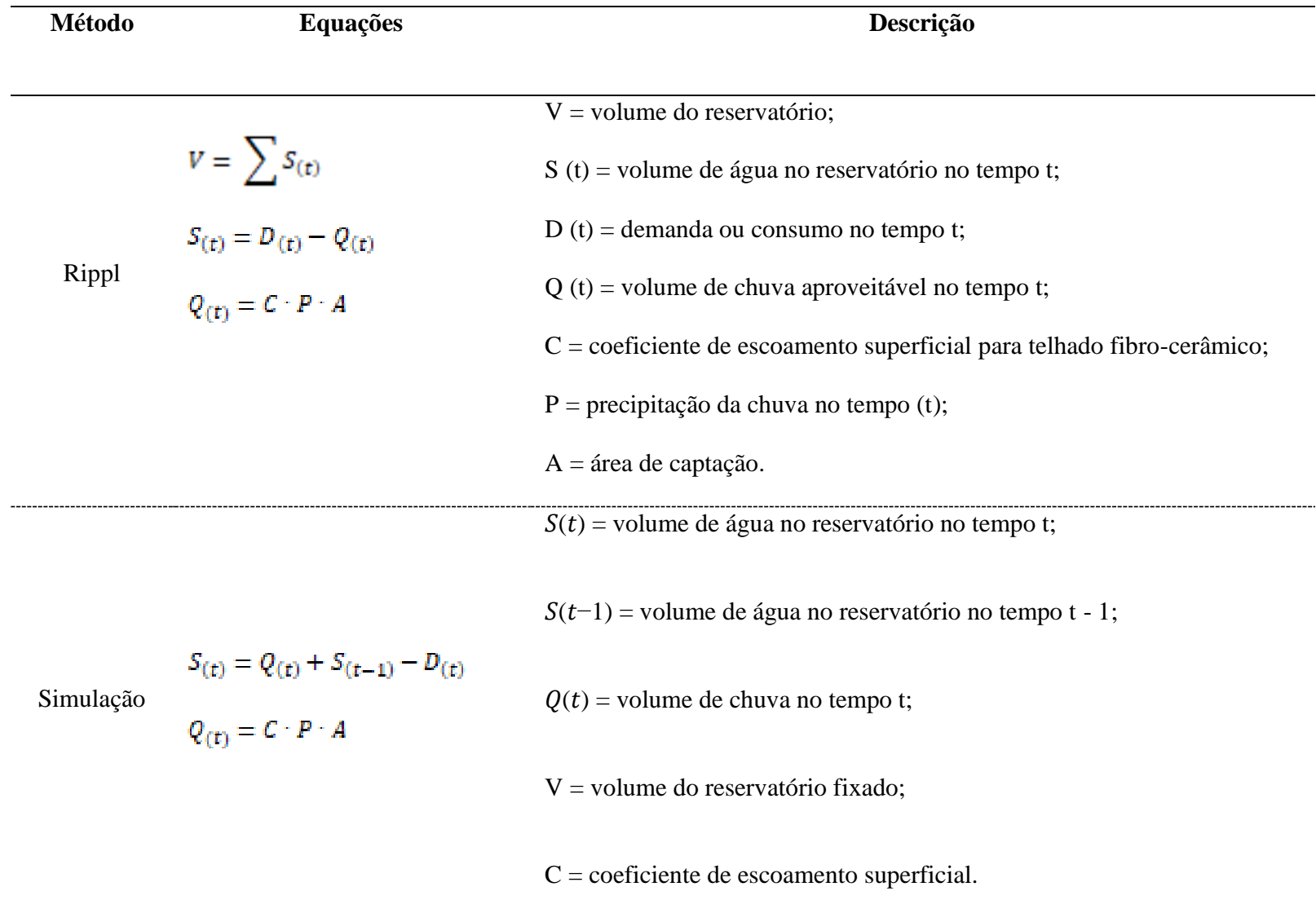


$\mathrm{P}=$ Valor numérico de precipitação média anual, expresso em milímetros $(\mathrm{mm})$;

Azevedo

$$
V=0,042 \cdot P \cdot A \cdot T
$$

Neto
$\mathrm{A}=$ Valor numérico da área de coleta em projeção, expresso em metros quadrados $\left(\mathrm{m}^{2}\right)$;

$\mathrm{T}=$ Valor numérico do número de meses de pouca chuva ou seca;

$\mathrm{V}=$ Valor numérico do volume de água aproveitável ou volume do reservatório, expresso em litros (L);

$\mathrm{V}=$ Valor numérico do volume aproveitável de água de chuva anual, expresso em litros (L);

Prático $V a=\min (V ; D)-0,06$

Alemão $\mathrm{D}=$ Valor numérico da demanda anual da água não potável, expresso em litros (L);

Vadotado $=$ Valor numérico do volume de água do reservatório, expresso em litros (L).

$\mathrm{V}=$ valor numérico do volume de água aproveitável e o volume de água

Prático

$$
V=0,05 \cdot P \cdot A
$$

Inglês

$\mathrm{P}=$ valor numérico da precipitação média anual, expressa em litros (L);

$\mathrm{A}=$ valor numérico da área de captação de coleta em projeção, expresso em metros quadrados $\left(\mathrm{m}^{2}\right)$.

$\mathrm{C}=$ coeficiente de escoamento superficial;

$\mathrm{P}=$ precipitação média mensal;

I = interceptação da água que molha as superfícies e perdas por

$$
Q=A \cdot C \cdot(P-I)
$$

evaporação, geralmente $2 \mathrm{~mm}$;

Prático australiano

$$
V_{(\mathrm{i})}=V_{(\mathrm{i} t-1)}+Q_{(\mathrm{d})}-D_{(\mathrm{d})}
$$

A é a área de coleta;

$\mathrm{Q}=$ volume mensal produzido pela chuva;

$\mathrm{V}(\mathrm{t})$ = volume de água que está no tanque no fim do mês t;

$\mathrm{V}(\mathrm{t}-1)$ = volume de água que está no tanque no início do mês t;

$\mathrm{Q}(\mathrm{t})=$ volume mensal produzido pela chuva no mês $\mathrm{t}$;

$\mathrm{D}(\mathrm{t})=$ demanda mensal.

Fonte: ABNT NBR 15527/2007.

Destaca-se que para aplicação dos métodos de Azevedo Neto, Prático Inglês e Prático Alemão, foram consideradas as precipitações médias anuais da série histórica. Além disso, 
foram considerados como meses de pouca chuva àqueles em que a precipitação mensal foi inferior a $70 \mathrm{~mm}$.

Para os métodos de Rippl, Prático Australiano e Simulação, foram consideradas as precipitações médias mensais da série histórica. Para aplicação dos métodos foi considerado que no primeiro mês o reservatório inicia-se vazio; o Coeficiente de Runnof de 0,8; e as perdas por interceptação/evapotranspiração foram de $2 \mathrm{~mm}$, conforme ABNT NBR $15527 / 2007$.

\section{Análise de viabilidade econômica}

Para os cálculos da redução do custo mensal, foi considerado um consumo médio aproximadamente $10 \%$ inferior ao recomendado para esses municípios pela GPE-NI-011-01 (2020), da COMPESA, por se tratar de uma edificação popular de baixo consumo. Assim, o valor adotado foi de $18 \mathrm{~m}^{3 /}$ mês, A implantação do sistema AAP provocou uma redução no consumo médio da água de 18 para $15 \mathrm{~m}^{3} / \mathrm{mês}$, redução de $3 \mathrm{~m}^{3} / \mathrm{mês}$ referente ao uso da descarga. Os cálculos foram realizados conforme os valores das tarifas cobradas pela COMPESA (2017), para unidades residenciais. O valor da tarifa mensal era de R\$40,18até $10 \mathrm{~m}^{3}$ e para cada $\mathrm{m}^{3}$ excedente era cobrado $\mathrm{R} \$ 4,61$ adicional até o limite de $20 \mathrm{~m}^{3}$. Portanto, o valor da tarifa de água seria de $\mathrm{R} \$ 77,06$ mensais $\left(18 \mathrm{~m}^{3}\right)$. Com a redução da demanda de 18 para $15 \mathrm{~m}^{3}$, a tarifa mensal reduz para $\mathrm{R} \$ 63,23$ economizando $\mathrm{R} \$ 13,83$ mensais.

Para os cálculos do valor de investimento do reservatório considerou-se o valor estimado por Tomaz (2003) para cisterna de concreto armado. Como o valor pode variar entre $\$ 107 / \mathrm{m}^{3}$ e $\$ 178 / \mathrm{m}^{3}$, optou-se por um valor médio de $\$ 142 / \mathrm{m}^{3}$. Considerando o valor médio do dólar para o ano de 2019 por volta de $\mathrm{R} \$ 3,95$, estabeleceu-se que o valor do metro cúbico para construção do reservatório seria de aproximadamente de $\mathrm{R} \$ 560,90$.

Desse modo, foi realizada uma análise da viabilidade econômica, a partir de um balanço entre o custo mensal de água e o custo de implantação do reservatório. Salienta-se que nesse estudo, não foi considerada a variabilidade do valor da moeda, bem como, a possível mudança das taxas de cobrança de água, por parte da companhia. 


\section{Resultados e Discussão}

\section{Cálculo do volume do reservatório}

Na Tabela 3 são apresentados os dados referentes ao cálculo do volume pelos métodos de Azevedo Neto e Prático Inglês. É possível notar que os volumes do reservatório determinados pelo método de Azevedo Neto variam proporcionalmente à precipitação média anual e ao número de meses de pouca chuva, dadas as condições de contorno do problema estudado, em que a área de captação é fixada.

Tabela 3 - Dados utilizados para o preenchimento das variáveis dos métodos Azevedo Neto e Prático Inglês.

\begin{tabular}{|c|c|c|c|c|c|}
\hline \multirow{2}{*}{ Estação } & \multirow{2}{*}{$\begin{array}{l}\text { Precipitação } \\
\text { Média Anual } \\
\text { (mm) }\end{array}$} & \multirow{2}{*}{$\begin{array}{c}\text { Número } \\
\text { Médio Anual } \\
\text { de Meses com } \\
\text { pouca chuva }\end{array}$} & \multirow{2}{*}{$\begin{array}{c}\text { Área de } \\
\text { Coleta }\left(\mathbf{m}^{2}\right)\end{array}$} & \multicolumn{2}{|c|}{$\begin{array}{l}\text { Volume Calculado do } \\
\text { Reservatório }\left(\mathbf{m}^{3}\right)\end{array}$} \\
\hline & & & & Azevedo Neto & $\begin{array}{l}\text { Prático } \\
\text { Inglês }\end{array}$ \\
\hline Bezerros & 440,6 & 9,5 & 160 & 28,13 & 3,52 \\
\hline Caruaru & 413,2 & 10,5 & 160 & 29,16 & 3,31 \\
\hline Gravatá & 571,5 & 8,5 & 160 & 32,64 & 4,57 \\
\hline
\end{tabular}

Fonte: Os Autores (2020).

Foi verificado que o maior volume calculado é para o município de Gravatá, com $32,64 \mathrm{~m}^{3}$, que se destaca por também apresentar maior média anual de precipitação $(571,5$ $\mathrm{mm})$. Já o menor volume calculado, foi verificado para o município de Bezerros $\left(28,13 \mathrm{~m}^{3}\right.$, correspondendo a 86,16\% do volume de Gravatá) que, apesar, de registrar média de precipitação superior ao município de Caruaru, apresenta um número de meses de pouca chuva inferior. Caruaru, com volume de $29,16 \mathrm{~m}^{3}$, corresponde a $89,31 \%$ do volume de Gravatá.

O método Prático Inglês, por sua vez, não faz consideração em relação a sazonalidade mensal de chuvas, o cálculo do volume do reservatório é diretamente proporcional à precipitação média anual e a área de captação. Portanto, ao considerar uma área de coleta fixa para os três municípios, aquele que apresentou o maior volume calculado foi o que registrou maior média de precipitação, ou seja, o município de Gravatá, com 4,57 $\mathrm{m}^{3}$, enquanto que Caruaru, que registrou a menor média de precipitação, registrou também o menor volume para o reservatório, de $3,31 \mathrm{~m}^{3}$. Percentualmente, o volume calculado por este método, para as 
Research, Society and Development, v. 9, n. 8, e409985102, 2020

(CC BY 4.0) | ISSN 2525-3409 | DOI: http://dx.doi.org/10.33448/rsd-v9i8.5102

cidades de Bezerros e Caruaru correspondem a 77,1\% e 72,3\% respectivamente comparado ao volume da cidade de Gravatá, que detém da maior precipitação média anual.

Para o cálculo do volume do reservatório pelo método pelo Prático Alemão, no qual os dados estão apresentados na Tabela 4, o volume necessário para o reservatório foi considerado o menor valor entre $6 \%$ da demanda anual de consumo e $6 \%$ do volume anual de precipitação captada.

Tabela 4 - Dados utilizados para o preenchimento das variáveis do método Prático Alemão para cada município.

\begin{tabular}{lccccccc}
\hline Estação & $\begin{array}{c}\text { Demanda } \\
\text { Mensal } \\
\left(\mathbf{m}^{3}\right)\end{array}$ & $\begin{array}{c}\text { Demanda } \\
\text { Anual - Vd } \\
\left(\mathbf{m}^{3}\right)\end{array}$ & $\begin{array}{c}\text { Precipitação } \\
\text { Média Anual } \\
(\mathbf{m m})\end{array}$ & $\begin{array}{c}\text { Área de } \\
\mathbf{C o l e t a} \\
\left(\mathbf{m}^{2}\right)\end{array}$ & $\begin{array}{c}\text { Volume } \\
\text { médio } \\
\text { anual de } \\
\mathbf{c h u v a}- \\
\mathbf{V p}\left(\mathbf{m}^{3}\right)\end{array}$ & $\begin{array}{c}\text { Mínimo } \\
\text { entre Vd e } \\
\mathbf{V p}\left(\mathbf{m}^{3}\right)\end{array}$ & $\begin{array}{c}\text { Volume } \\
\text { Calculado do } \\
\text { Reservatório } \\
\left(\mathbf{m}^{3}\right)\end{array}$ \\
\hline Bezerros & 3 & 36 & 440,6 & 160 & 56,4 & 36,0 & 2,2 \\
Caruaru & 3 & 36 & 413,2 & 160 & 52,9 & 36,0 & 2,2 \\
Gravatá & 3 & 36 & 571,5 & 160 & 73,2 & 36,0 & 2,2 \\
\hline
\end{tabular}

Fonte: Os Autores (2020).

Para todos os casos, em virtude de o cenário considerar o consumo de água e área de captação fixados, a demanda anual foi menor que a precipitação anual, resultando em um mesmo volume para as três cidades $\left(2,2 \mathrm{~m}^{3}\right)$, isto é, $6 \%$ da demanda anual de águas pluviais. $\mathrm{Na}$ Tabela 5 são apresentados os valores obtidos para o volume de reservatório pelo método de Rippl.

Tabela 5 - Dados utilizados para o preenchimento das variáveis do método de Rippl.

\begin{tabular}{lcccccc}
\hline \multirow{2}{*}{ Estação } & $\begin{array}{c}\text { Chuva Média } \\
\text { Anual (mm) }\end{array}$ & $\begin{array}{c}\text { Área de } \\
\text { Captação } \\
\left(\mathbf{m}^{2}\right)\end{array}$ & $\begin{array}{c}\text { Demanda } \\
\text { Anual } \\
\left(\mathbf{m}^{3}\right)\end{array}$ & $\begin{array}{c}\text { Volume de } \\
\text { chuva } \\
\text { anual }\left(\mathbf{m}^{3}\right)\end{array}$ & $\begin{array}{c}\mathbf{S}(\mathbf{t})=\mathbf{D}(\mathbf{t}) \\
-\mathbf{Q}(\mathbf{t})\end{array}$ & $\begin{array}{c}\text { Volume } \\
\mathbf{V}=\sum \mathbf{S}_{(\mathbf{t})} \\
\text { para } \mathbf{S}(\mathbf{t})>\mathbf{0}\end{array}$ \\
\hline Bezerros & 440,6 & 160 & 36 & 56,4 & $-20,4$ & 5,2 \\
Caruaru & 413,2 & 160 & 36 & 52,9 & $-16,9$ & 1,9 \\
Gravatá & 571,5 & 160 & 36 & 73,1 & $-37,1$ & 2,1 \\
\hline
\end{tabular}

Fonte: Os Autores (2020).

Para o município de Bezerros, os meses de janeiro, de março a julho e dezembro apresentaram balanço negativo, ou seja, nesses meses o volume de chuva aproveitável foi maior que a demanda requerida. Portanto, para esses meses, não seria necessário armazenar 
águas pluviais, conforme o método. Já nos meses de fevereiro e de agosto a novembro, cujos balanços foram positivos, com demanda superior ao volume de chuva aproveitável, o somatório dos volumes resultantes foi considerado para determinar o volume necessário do reservatório. A cisterna registrou um volume igual a 5,2 $\mathrm{m}^{3}$.

O município de Caruaru registrou um balanço negativo para os meses de março a julho, novembro e dezembro. Os meses de janeiro e fevereiro apresentaram balanço igual a zero, ou seja, com demanda igual ao volume de águas pluviais aproveitáveis. Contudo, os meses de agosto a outubro apresentaram balanço positivo, cujo somatório resultou num volume de reservatório igual a $1,9 \mathrm{~m}^{3}$.

Por fim, para o município de Gravatá, apenas os meses de setembro e novembro apresentaram balanços positivos, resultando em um volume total de $2,1 \mathrm{~m}^{3}$ para o reservatório de águas pluviais.

Apesar de Caruaru ser o município a registrar menor média mensal de chuvas, é o que apresenta menor volume estimado para o reservatório. Acredita-se que isso se justifica pela regularidade do regime pluviométrico ao longo do ano desse município, com volume de chuva suprindo a demanda em até 7 meses, e, além disso, nos meses que não a demanda não é suprida, o déficit hídrico não é tão elevado. Na comparação com Bezerros, a demanda é suprida na mesma quantidade de meses que Caruaru, porém, os déficits são maiores, por exemplo: só no mês de novembro, cujo déficit foi de $2,3 \mathrm{~m}^{3}$, já supera o acumulado do município de Caruaru (1,9 m³). O mesmo pode ser observado no balanço geral para cidade de Gravatá.

A determinação do volume do reservatório de água pluvial pelo método Prático Australiano consistiu num processo iterativo, em que foram fixados diferentes volumes e calculados os meses em que o reservatório atende ou não a demanda de projeto. Além disso, calculou-se a probabilidade de falha e a confiança dos volumes avaliados para o reservatório.

Na Tabela 6 são apresentados os volumes de reservatório avaliados para cada município, com suas respectivas probabilidades de falha e confianças. Recomenda-se que o volume do reservatório escolhido possua valores de confiança entre 90 e $99 \%$. 
Tabela 6 - Resultados do método Prático Australiano para cidades de Bezerros, Caruaru e Gravatá.

\begin{tabular}{|c|c|c|c|}
\hline $\begin{array}{c}\text { Volume do } \\
\text { Reservatório } \\
\qquad\left(\mathbf{m}^{3}\right)\end{array}$ & $\begin{array}{l}\text { N}^{0} \text { de Meses que o } \\
\text { Reservatório não } \\
\text { atende à demanda }\end{array}$ & $\begin{array}{c}\text { Probabilidade } \\
\text { de Falha }\end{array}$ & Confiança \\
\hline \multicolumn{4}{|c|}{ Município: Bezerros } \\
\hline 6 & 1 & $8 \%$ & $92 \%$ \\
\hline 5 & 2 & $17 \%$ & $83 \%$ \\
\hline 4 & 2 & $17 \%$ & $83 \%$ \\
\hline 3 & 3 & $25 \%$ & $75 \%$ \\
\hline \multicolumn{4}{|c|}{ Município: Caruaru } \\
\hline 4 & 2 & $17 \%$ & $83 \%$ \\
\hline 3 & 2 & $17 \%$ & $83 \%$ \\
\hline 2 & 4 & $33 \%$ & $67 \%$ \\
\hline 1 & 5 & $42 \%$ & $58 \%$ \\
\hline \multicolumn{4}{|c|}{ Município: Gravatá } \\
\hline 3 & 0 & $0 \%$ & $100 \%$ \\
\hline 2 & 1 & $8 \%$ & $92 \%$ \\
\hline 1 & 1 & $8 \%$ & $92 \%$ \\
\hline 0,3 & 3 & $25 \%$ & $75 \%$ \\
\hline
\end{tabular}

Fonte: Os Autores (2020).

É possível observar que, para Bezerros, utilizando o volume de $6 \mathrm{~m}^{3} \mathrm{em}$ apenas um mês o reservatório precisaria de suprimento de água externo para atender a demanda, com confiança de $92 \%$. Para o município de Caruaru, um reservatório de volume 4 mé suficiente para que o atendimento à demanda seja satisfeito em 10 meses no ano. Não se conseguiu valores de reservatório que obtivessem confiança maior que $90 \%$, aumentando o volume do reservatório continuaríamos com esses dois meses necessitando de suprimento, o que implicaria apenas no aumento dos custos de construção de reservatório. Uma possível solução para o caso de Caruaru seria o aumento da área de captação. Em relação ao município de Gravatá, foi determinado que para um volume de reservatório de $3 \mathrm{~m}^{3}$, não haveria necessidade de suprimento externo em nenhum mês, com 100\% de confiança.

Para o método da Simulação a ABNT NBR 15527/2007 estabelece a hipótese de reservatórios cheios no início da análise. Entretanto, para esse trabalho, foi considerado o reservatório vazio, em uma situação de primeiro uso. O cálculo foi processado considerando 
Research, Society and Development, v. 9, n. 8, e409985102, 2020

(CC BY 4.0) | ISSN 2525-3409 | DOI: http://dx.doi.org/10.33448/rsd-v9i8.5102

os dados de precipitações médias mensais, no qual foram realizadas simulações para diversos valores de volume, até atingir um valor em que, aumentar a dimensão do reservatório não resultaria em um aumento no atendimento da demanda.

Na Tabela 7 são apresentadas as simulações com volumes, probabilidade de falha e confiança para os municípios de Bezerros, Caruaru e Gravatá.

Tabela 7 - Resultados do Método da simulação para cidades de Bezerros, Caruaru e Gravatá.

\begin{tabular}{|c|c|c|c|}
\hline $\begin{array}{c}\text { Volume do } \\
\text { Reservatório }\left(\mathbf{m}^{3}\right)\end{array}$ & $\begin{array}{l}\mathrm{N}^{0} \text { de Meses que o } \\
\text { Reservatório não } \\
\text { atende à demanda }\end{array}$ & $\begin{array}{c}\text { Probabilidade de } \\
\text { Falha }\end{array}$ & Confiança \\
\hline \multicolumn{4}{|c|}{ Município: Bezerros } \\
\hline 5 & 0 & $0 \%$ & $100 \%$ \\
\hline 4 & 1 & $8 \%$ & $92 \%$ \\
\hline 3 & 1 & $8 \%$ & $92 \%$ \\
\hline 2 & 2 & $17 \%$ & $83 \%$ \\
\hline \multicolumn{4}{|c|}{ Município: Caruaru } \\
\hline 3 & 0 & $0 \%$ & $100 \%$ \\
\hline 2 & 0 & $0 \%$ & $100 \%$ \\
\hline 1 & 1 & $8 \%$ & $92 \%$ \\
\hline 0,5 & 2 & $17 \%$ & $83 \%$ \\
\hline \multicolumn{4}{|c|}{ Município: Gravatá } \\
\hline 3 & 0 & $0 \%$ & $100 \%$ \\
\hline 2 & 1 & $8 \%$ & $92 \%$ \\
\hline 1 & 1 & $8 \%$ & $92 \%$ \\
\hline 0,3 & 1 & $8 \%$ & $92 \%$ \\
\hline
\end{tabular}

Fonte: Os Autores (2020).

Para Bezerros, a partir de $3 \mathrm{~m}^{3}$ já se consegue uma confiança de $92 \%$ de atendimento à demanda e com $5 \mathrm{~m}^{3}$ obtém-se confiança de $100 \%$ no atendimento. Em Caruaru se consegue níveis de confiança elevados com volumes pequenos, isto é, com $2 \mathrm{~m}^{3}$ de água já se obtém confiança de $100 \%$. E em Gravatá, o reservatório de $0,5 \mathrm{~m}^{3}$ a confiança está na casa dos $92 \%$, adquirindo uma confiança de $100 \%$ a partir de $3 \mathrm{~m}^{3}$.

Em suma, na Tabela 8 são apresentados todos os volumes de reservatórios obtidos pelos métodos propostos pela NBR 15527/2007. 
Research, Society and Development, v. 9, n. 8, e409985102, 2020

(CC BY 4.0) | ISSN 2525-3409 | DOI: http://dx.doi.org/10.33448/rsd-v9i8.5102

Tabela 8 - Resumo dos volumes de reservatório obtidos pelos métodos da NBR $15527 / 2007$.

\begin{tabular}{ccccccc}
\hline Estação & $\begin{array}{c}\text { Azevedo } \\
\text { Neto }\end{array}$ & $\begin{array}{c}\text { Prático } \\
\text { Inglês }\end{array}$ & $\begin{array}{c}\text { Prático } \\
\text { Alemão }\end{array}$ & Rippl & $\begin{array}{c}\text { Prático } \\
\text { Australiano* }\end{array}$ & Simulação* \\
\hline Bezerros & 28,1 & 3,5 & 2,2 & 5,2 & 6,0 & 5,0 \\
Caruaru & 29,2 & 3,3 & 2,2 & 2,9 & 4,0 & 2,0 \\
Gravatá & 32,6 & 4,6 & 2,2 & 2,1 & 3,0 & 3,0 \\
\hline
\end{tabular}

Nota: Para os métodos Prático Australiano e da Simulação foram apresentados os volumes que resultaram na maior confiança. Fonte: Os Autores (2020).

Os resultados obtidos no dimensionamento dos reservatórios de águas pluviais, calculados a partir dos métodos preconizados na NBR 15527/2007, apresentaram consideráveis discrepâncias de volumes para o cenário proposto, residência unifamiliar com demanda e área de captações fixadas.

Os três municípios, apesar de estarem inseridos na mesma bacia hidrográfica do Ipojuca e possuírem regimes pluviométricos semelhantes, apresentam reservatórios com volumes compreendidos entre $2 \mathrm{~m}^{3}$ e $32 \mathrm{~m}^{3}$, uma diferença volumétrica de mais de $1500 \%$. Entretanto, a variação nos resultados dos diferentes métodos também foi ressaltada na literatura,tanto em estudos que consideraram cenários com incrementos na área de captação de água e do consumo requerido (Brandão\& Marcon, 2018), quanto os estudos que consideraram diferentes regimes pluviométricos no Brasil (Fonseca\& Menezes Filho, 2019; Dornelles et al., 2010).

Os métodos Azevedo Neto, Prático Inglês e Prático Alemão possuem como característica comum não considerar o balanço hídrico dentro do reservatório para o cálculo do volume. Este é determinado a partir do número máximo de dias necessários para que a água fique armazenada, sendo sensível apenas ao regime pluviométrico da região e à área da superfície de captação. Os volumes calculados pelo método de Azevedo Neto foram os maiores valores encontrados, variando entre 28,1 e 32,6m³. Já o método prático inglês, que assim como o de Azevedo Neto é calculado por meio de equação direta, resultou em valores muito menores variando entre 3,31 e $4,57 \mathrm{~m}^{3}$. Essa diferença se deve ao fato de que o volume calculado por Azevedo Neto é diretamente proporcional ao número de meses de pouca chuva, os quais, no caso em apreço, superiores a 8 meses, são considerados elevados na mesorregião do agreste pernambucano. Já o método Prático alemão resultou em valores iguais para as três cidades, uma vez que o cenário considerado não prevê alterações na área de captação ou na 
demanda de água. Sendo assim, já que a demanda $\left(36 \mathrm{~m}^{3}\right)$ nos três casos é menor que a oferta anual, o volume será $6 \%$ desse volume invariavelmente.

Os resultados obtidos por Fonseca \& Menezes Filho (2019) para as cidades de Belo Horizonte e Goiânia apresentam volumes de reservatório na mesma ordem de grandeza aos determinados nesse estudo pelos métodos Azevedo Neto, Prático Inglês e Prático Alemão. O autor ressalta que se tratando de residências familiares, volumes superiores à dezena são muito elevados e o espaço ocupado por reservatórios dessa magnitude pode ser um obstáculo considerável.

Entre os métodos de dimensionamento que consideram o balanço hídrico do sistema estão o método de Rippl, o Prático Australiano e da simulação. O método de Rippl apresentou valores de reservatório suficientes para suprir a demanda por água integralmente no balanço mensal, cujas diferenças volumétricas associadas ao déficit hídrico do regime pluviométrico de cada município. Nesse contexto, as cisternas resultaram em uma maior diferença para cidade de Bezerros com quase 5m, enquanto Caruaru e Gravatá tiveram 2,9 e 2,1 $\mathrm{m}^{3}$ respectivamente. Segundo Brandão e Marcon (2018), o método de Rippl Mensal regulariza a vazão para suprir a demanda de água no período mais seco da série histórica, o que lhe garante determinar volumes de reservatório com alta garantia de abastecimento.

O método Prático Australiano e o método da simulação podem ser avaliados em função de confiança associada ao volume do reservatório, cujos valores determinados acusam confiança entre 90 e 99\%. O método Prático Australiano, entretanto, por fazer uso das médias pluviométricas mensais plurianuais, tem sua precisão dos resultados menor se comparada ao método da Simulação. Fonseca \& Menezes Filho (2019) afirmam que, apesar de dispender de grande tempo, a simulação fornece resultados mais seguros e confiáveis.

\section{Viabilidade Econômica}

Na Tabela 9 é apresentada a estimativa de retorno do valor investido na construção do reservatório com a economia da conta de água. Os tempos de retorno estimados, com exceção do método Azevedo Neto, variam de 20,3 a 6,8 anos, garantindo benefícios financeiros em médio prazo. O método Azevedo Neto, conforme já mencionado, além de determinar volumes que requerem espaços desproporcionais à unidade familiar, apresentam elevados custo de investimento e tempo de retorno inviáveis, superiores a 90 anos. 
Research, Society and Development, v. 9, n. 8, e409985102, 2020

(CC BY 4.0) | ISSN 2525-3409 | DOI: http://dx.doi.org/10.33448/rsd-v9i8.5102

Tabela 9 - Valor do investimento no reservatório e estimativa do tempo de retorno financeiro por método.

\begin{tabular}{|c|c|c|c|c|c|c|}
\hline \multirow[b]{2}{*}{ Método } & \multicolumn{2}{|c|}{ Bezerros } & \multicolumn{2}{|c|}{ Caruaru } & \multicolumn{2}{|c|}{ Gravatá } \\
\hline & Investimento & $\begin{array}{c}\text { Tempo } \\
\text { de retorno } \\
\text { (anos) }\end{array}$ & Investimento & $\begin{array}{c}\text { Tempo de } \\
\text { retorno } \\
\text { (anos) }\end{array}$ & Investimento & $\begin{array}{c}\text { Tempo de } \\
\text { retorno } \\
\text { (anos) }\end{array}$ \\
\hline Azevedo Neto & $\mathrm{R} \$ 15.761,29$ & 95,0 & $\mathrm{R} \$ 16.378,28$ & 98,7 & $\mathrm{R} \$ 18.285,34$ & 110,2 \\
\hline Prático Inglês & $\mathrm{R} \$ 1.963,15$ & 11,8 & $\mathrm{R} \$ 1.850,97$ & 11,2 & $\mathrm{R} \$ 2.580,14$ & 15,5 \\
\hline $\begin{array}{l}\text { Prático } \\
\text { Alemão }\end{array}$ & $\mathrm{R} \$ 1.233,98$ & 7,4 & $\mathrm{R} \$ 1.233,98$ & 7,4 & $\mathrm{R} \$ 1.233,98$ & 7,4 \\
\hline Rippl & $\mathrm{R} \$ 2.916,68$ & 17,6 & $\mathrm{R} \$ 1.626,61$ & 9,8 & $\mathrm{R} \$ 1.177,89$ & 7,1 \\
\hline $\begin{array}{c}\text { Prático } \\
\text { Australiano }\end{array}$ & $\mathrm{R} \$ 3.365,40$ & 20,3 & $\mathrm{R} \$ 2.243,60$ & 13,5 & $\mathrm{R} \$ 1.682,70$ & 10,1 \\
\hline Simulação & $\mathrm{R} \$ 2.804,50$ & 16,9 & $\mathrm{R} \$ 1.121,80$ & 6,8 & $\mathrm{R} \$ 1.682,70$ & 10,1 \\
\hline
\end{tabular}

Fonte: Os Autores (2020).

Lima et al. (2017) estimaram um tempo de retorno do investimento para implantação de um reservatório de água de $50 \mathrm{~m}^{3}$ numa escola pública no Recife em 6 anos e 25 dias.O tempo consideravelmente menor se deve ao fato de que a estimativa do consumo de água para fins não potáveis da escola possui um potencial de economia de água potável da ordem de $59,6 \%$, em comparação com os estimados $16,7 \%$ do gerado no cenário considerado nesse estudo.

\section{Considerações Finais}

Cada um dos métodos apresenta suas particularidades, permitindo ao tomador de decisões levar considerar as características de seu projeto, assim como as limitações financeiras e os benefícios do uso racional da água. Mediante isso, é interessante que se escolha o volume cujo cálculo levou em consideração a demanda de água necessária para suprir as necessidades, além de um custo-benefício esperado pelo consumidor.

Os métodos da Simulação e Prático Australiano são os mais indicados pelo fato de além de haver a consideração oferta/demanda, ambos também oferecem uma taxa de confiança quanto ao atendimento à demanda, além de volumes de reservatório que não devem gerar custos de implantação exagerados. Ambos também têm estimativa de retorno do valor investido relativamente rápido, comparado a outros métodos. 
Research, Society and Development, v. 9, n. 8, e409985102, 2020

(CC BY 4.0) | ISSN 2525-3409 | DOI: http://dx.doi.org/10.33448/rsd-v9i8.5102

Como sugestões de trabalhos futuros, recomenda-se a análise em conformidade com as condicionantes do sistema de abastecimento de água nos municípios. Ou seja, aplicar os métodos para os meses em que o abastecimento não esteja sendo realizado.

\section{Agradecimentos}

O presente trabalho foi realizado com apoio da Coordenação de Aperfeiçoamento de Pessoal de Nível Superior - Brasil (CAPES), processo No 88882.379365/2019-01, do projeto “Transferência de Água e Misturas de Poluentes Reativos em Solos Antropizados” (processo CNPq no 436875 / 2018-7) e do projeto “Observatório Nacional de Dinâmica da Água e do Carbono na Caatinga Bioma ONDACBC (processo CNPq nº 465764 / 2014-2; processo CAPES nº 88887.136369 / 2017-00; processo FACEPE APQ-0498-3.07 / 17).

\section{Referências}

APAC. (2017). Agência Pernambucana de Águas e Clima. Disponível em: http://www.apac.pe.gov.br.

Brandão, J. L. B., \& Marcon, P. (2018). Análise dos métodos de dimensionamento de reservatórios de águas pluviais sugeridos pela NBR 15527/2007 com base na simulação diária. EngSanit Ambient, 23(6): 1031-41.

Campisano, A., Butler, D., Ward, S., Burns, M. J., Friedler, E., DeBusk, K., \& Han, M. (2017). Urban rainwater harvesting systems: Research, implementation and future perspectives. Waterresearch, 115: 195-209.

Campisano, A., Di Liberto, D., Modica, C., \&Reitano, S. J. P. E. (2014). Potential for peak flow reduction by rainwater harvesting tanks. Procedia Engineering, 89: 1507-1514.

Compesa (2017). Tarifas de cobrança de água. Portal eletrônico. Disponível em: https://lojavirtual.compesa.com.br:8443/gsan/exibirConsultarEstruturaTarifariaPortalAction.d o. 
Dornelles, F., Tassi, R., \& Goldenfum, J. A. (2010). Avaliação das técnicas de dimensionamento de reservatórios para aproveitamento de água de chuva. Revista Brasileira de Recursos Hídricos, 15(2): 59-68.

Duarte, G. W., Mattos, A. S., Eing, C., da Silva, D. L., Frasson, K. C., \&Eckert, C. L. (2015). Aproveitamento de água pluvial para fins não potáveis: estudo de caso na jardinagem. Revista Ciência \& Cidadania, 1(1).

Feitosa, E. R., Yada, M. M., \& Soares, N. M. (2018). Uso de cisternas na captação da água da chuva para uso animal. Revista Interface Tecnológica, 15(1): 305-314.

Fonseca, V. L. A. \& Menezes Filho, C. M. (2019). Estudo comparativo entre métodos de dimensionamento para reservatórios de sistemas de aproveitamento de águas pluviais. Holos Environment, 19 (2): 287-303.

Fonseca, T. D. dos S., Júnior, L. U. R., \& Faria, J. P. R. (2017). Estudo da viabilidade econômica do aproveitamento de água de chuva para fins não potáveis em residências em Itajubá-Minas Gerais. Revista Científica da FEPI-Revista Científic@ Universitas, 4(1).

Haque, M. M., Rahman, A., \& Samali, B. (2016). Evaluation of climate change impacts on rainwater harvesting. Journal of Cleaner Production, 137: 60-69.

GPE, G. D. P. (2020). Norma internade engenharia. Norma, 21, 08. Companhia Pernambucana de Saneamento - COMPESA.

Kuchinski, V., \& Gastaldini, M. D. C. C. (2016). Viabilidade técnica e econômica do aproveitamento das águas de chuva e cinza para consumo não potável em edifício residencial de Santa Maria (RS).

Lee, K. E., Mokhtar, M., Hanafiah, M. M., Halim, A. A., \&Badusah, J. (2016). Rainwater harvesting as an alternative water resource in Malaysia: potential, policies and development. Journal of Cleaner Production, 126, 218-222. 
Lima, K. L. B. de A., Nunes, L. G. C. F., \& da Silva, S. R. (2017). Análise da viabilidade de implantação de captação de águas pluviais em escola pública no Recife-PE. Revista Nacional de Gerenciamento de Cidades, 5(36).

Moura, M. R. F. de, da Silva, S. R., \& do Rêgo Barros, E. X. (2018). Análise de implantação de um sistema de aproveitamento de água pluvial em um empreendimento residencial na cidade de Recife-PE. Tecno-Lógica, 22(1): 66-72.

NBR, A. (2007). 15527: Água de chuva-aproveitamento de coberturas em áreas urbanas para fins não potáveis-Requisitos. Associação Brasileira de Normas Técnicas. Rio de Janeiro, 8.

Neves Filho, A. O., Pereira, D. F., Mendes, L. F., Almeida, M. S. T., Borges, T. J., \& de Melo, M. C. (2019). Potencial de aproveitamento de água pluvial em áreas urbanas na bacia do rio verde. Semioses, 13(2), 28-42.

Pelak, N., \& Porporato, A. (2016). Sizing a rainwater harvesting cistern by minimizing costs. Journal of hydrology, 541, 1340-1347.

Palla, A., Gnecco, I., \& La Barbera, P. (2017). The impact of domestic rainwater harvesting systems in storm water runoff mitigation at the urban block scale. Journalofenvironmental management, 191: 297-305.

Pereira, F. V. de S., Mendes, R. L. R., Fernandes, L. L., Bello, L. A. L., \& do Rosário Duarte, C. K. A. (2018). Potencial do aproveitamento de água de chuva nos municípios de Curuçá e Igarapé Açu no estado do Pará. Revista Brasileira de Gestão Ambiental, 12(4), 07-14.

Pereira A. S. et al. (2018). Metodologia da pesquisa científica. [e-book]. Santa Maria. Ed. UAB/NTE/UFSM. Disponível em:

https://repositorio.ufsm.br/bitstream/handle/1/15824/Lic_Computacao_MetodologiaPesquisa-Cientifica.pdf?sequence $=1$.

Souza, J. F., Neto, M. R. F., Souza, M. A. S., \&Veneu, D. M. (2016). Aproveitamento de água de chuva para usos não potáveis na Universidade Severino Sombra. Revista Eletrônica TECCEN, 9(1): 35-46. 
Tomaz, P. (2003). Aproveitamento de água de chuva. São Paulo. Navegar Editora (2): 180.

Teston, A., Geraldi, M. S., Colasio, B. M., \&Ghisi, E. (2018). Rainwater harvesting in buildings in Brazil: A literature review. Water, 10(4): 471

\title{
Porcentagem de contribuição de cada autor no manuscrito
}

\author{
Lucas Vinícius dos Santo Ferreira - 25\% \\ José Martins de França Neto - 15\% \\ Larissa Virgínia da Silva Ribas - 15\% \\ Lucas Ravellys Pyrrho de Alcântara - 10\% \\ Severino Martins dos Santos Neto - $10 \%$ \\ Antonio Celso Dantas Antonino - 5\% \\ Artur Paiva Coutinho - 20\%
}

